\title{
Hardware Memory Management for Future Mobile Hybrid Memory Systems
}

\author{
Fei Wen \\ feitamu@gmail.com \\ Mian Qin
celery1124@tamu.edu \\ Department of Electrical \& Computer Engineering \\ Texas A\&M University
}

\author{
Narasimha Reddy \\ reddy@tamu.edu
}

\begin{abstract}
The current mobile applications have rapidly growing memory footprints, posing a great challenge for memory system design. Insufficient DRAM main memory will incur frequent data swaps between memory and storage, a process that hurts performance, consumes energy and deteriorates the write endurance of typical flash storage devices. Alternately, a larger DRAM has higher leakage power and drains the battery faster. Further, DRAM scaling trends make further growth of DRAM in the mobile space prohibitive due to cost. Emerging nonvolatile memory (NVM) has the potential to alleviate these issues due to its higher capacity per cost than DRAM and minimal static power. Recently, a wide spectrum of NVM technologies, including phase-change memories (PCM), memristor, and 3D XPoint have emerged. Despite the mentioned advantages, NVM has longer access latency compared to DRAM and NVM writes can incur higher latencies and wear costs. Therefore integration of these new memory technologies in the memory hierarchy requires a fundamental rearchitecting of traditional system designs. In this work, we propose a hardware-accelerated memory manager (HMMU) that addresses both types of memory in a flat space address space. We design a set of data placement and data migration policies within this memory manager, such that we may exploit the advantages of each memory technology. By augmenting the system with this HMMU, we reduce the overall memory latency while also reducing energy consumption and writes to the NVM. Experimental results show that our design achieves a $39 \%$ reduction in energy consumption with only a $12 \%$ performance degradation versus an all-DRAM baseline that is likely untenable in the future.
\end{abstract}

\section{Introduction}

As the demand for mobile computing power scales, mobile applications with ever-larger memory footprints are being developed, such as high-resolution video decoding, high-profile games, etc. This trend creates a great challenge for current memory and storage system design. The historical approach to address memory footprints larger than the DRAM available is for the OS to swap less used pages to storage, keeping higher locality pages in memory. Given the latencies of modern storage systems (even "high" performance SSDs [12,16,39]) are several orders of magnitude higher than DRAM.However, allowing any virtual memory swapping to storage implies incurring a severe slowdown. Thus mobile device manufacturer rapidly expanded the DRAM size for the worst case possible memory footprint. For example, the DRAM capacity of the flagship phones from the Samsung Galaxy S series have expanded by $16 \mathrm{X}$ over the past ten years. While this approach has been largely successful to date, the size of DRAM is constrained by both cost/economics and energy consumption. Unlike data centers, mobile devices are highly cost-sensitive and have a highly limited energy budget. Moreover, the DRAM technology has a substantial background power, constantly consuming energy even in idle due to its periodic refresh requirement, which scales with DRAM capacity. Therefore a larger DRAM means a higher power budget and a shorter battery life, particularly given recent hard DRAM VLSI scaling limits. The approach of provisioning more DRAM is not sustainable and hard limits will soon be hit on the scaling of the future mobile memory system.

The emergence of several Non-Volatile-Memory (NVM) technologies, such as Intel 3D Xpoint [13], memristor [7], Phase-change-memory(PCM) [30], provides a new avenue to address this growing problem. These new memory devices promise an order of magnitude higher density [4] per cost and lower static power consumption than traditional DRAM technologies, however, their access delay is significantly higher, typically also within one order of magnitude of DRAM. Further, these new technologies show significant overheads associated with writes and are non-volatile. Thus, these emerging memory technologies present a unique opportunity to address the problems of growing application workload footprints with hybrid memory systems composed of both DRAM and emerging NVM memories. To exploit these new memory devices effectively, however, we must carefully consider their perfor- 
Slowdown vs. No Swap

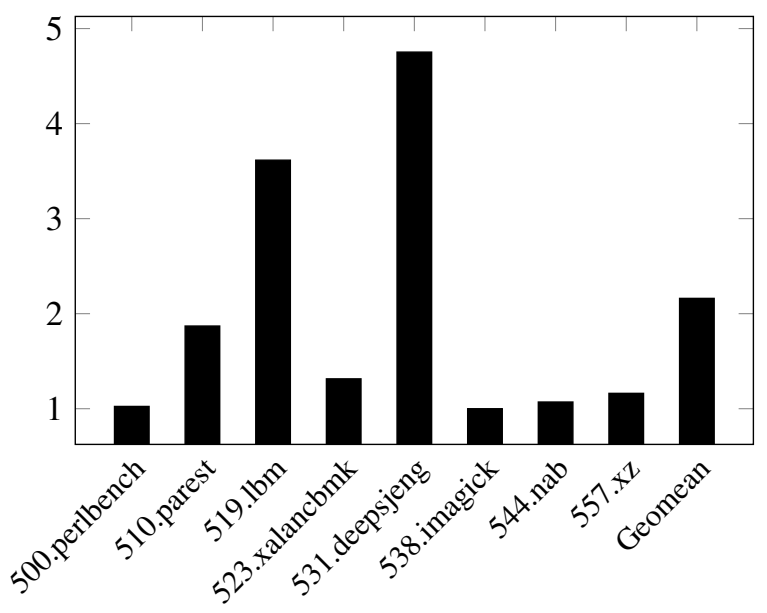

Figure 1: Performance Impact of OS Memory Management.

mance characteristics relative to existing points in the memory hierarchy. In particular, while memory access and movement in prior storage technologies such as flash and magnetic disk is slow enough that software management via the OS was feasible. With emerging NVM memory accesses at within an order of magnitude of DRAM, relying on traditional OS memory management techniques for managing placement between DRAM and NVM is insufficient as illustrated in Figure 1.

In Figure 1, a subset of benchmarks from the SPEC CPU2017 benchmark suite are executed in a system where around $128 \mathrm{MB}$ of the application's memory footprint is able to fit in the system DRAM directly. A ramdisk based swap file is set up to hold the remainder of the application memory footprint. Since this ramdisk swapfile is implemented in DRAM it represents an upper bound on the performance for pure software swapping. The results shown are normalized against a system where sufficient DRAM is available to capture the entire memory footprint. As we see, in this arrangement, the cost of pure OS managed swapping to NVM would be quite high, with applications seeing an average of $\sim 2 \mathrm{X}$ slowdown versus baseline. As we will show, a significant fraction of this overhead comes explicitly from the costs of the required page fault handling.

Some existing work has begun to explore system design for emerging hybrid memories. Broadly this prior work falls into one of two categories, first, some advocate using DRAM as a pure hardware managed cache for NVM [5, 29]. This approach implies a high hardware cost for metadata management and imposes significant capacity and bandwidth constraints. Second, some have advocated for a purely software, OS managed approach $[9,10,36]$. As we discussed previously, this approach implies significant slowdowns due to software overhead of the operating system calls.

Here we propose a new, hardware managed hybrid memory management scheme which retains the performance benefits of caching, without the high metadata overhead such an approach implies. Compared to previous work, our project has the following advantages:

- With a ratio of $1 / 8$ DRAM vs $7 / 8 \mathrm{NVM}$, we achieved $88 \%$ of the performance of an untenable full DRAM configuration, while reducing the energy consumption by $39 \%$.

- Compared to inclusive DRAM caches, we preserve the full main memory capacity for the user applications.

- Parallel access to both the DRAM and NVM is supported, rendering a higher effective memory bandwidth. This also helps to suppress the excessive cache insertion/replacements and prevent cache thrashing.

- The data placement and migration are executed by hardware. This eliminates the long latency incurred by the OS managed virtual memory swap process.

- Memory management and allocation are performed with a combination of page and sub-page-block sizes to ensure the best utilization of the available DRAM and to reduce the number and impact of writes to the NVM.

\section{Background and Motivation}

With emerging non-volatile memory technologies providing more memory system capacity, density, and lower static power, they have the potential to meet the continuously increasing memory usage of mobile applications. Given their different characteristics from traditional DRAM and storage, however, the design of systems comprising these new technologies together with traditional DRAM and storage is an open question. Here we examine the characteristics of these new memory technologies and the existing proposals to date on how to leverage them in system designs.

\subsection{Nonvolatile Memory Technology Charac- teristics}

Table 1 shows the relative characteristics of several emerging non-volatile memory technologies against traditional DRAM and storage [3, 25, 31]. While HDD and Flash have 100k and $2 \mathrm{k}$ times larger read access latency than DRAM respectively, the emerging NVM technologies have read access latencies typically within one order of magnitude of DRAM. Meanwhile emerging non-volatile memory technologies provide higher memory system capacity, density and lower static power.

Given their different characteristics from traditional DRAM and storage, however, the design of systems comprising these new technologies together with traditional DRAM and storage is an open question. Here we examine the characteristics of these new memory technologies and the existing proposals to date on how to leverage them in system designs. 
Table 1: Approximate Performance Comparison of Different Memory Technologies [3, 25, 38]

\begin{tabular}{c|c|c|c|c|c|c}
\hline Technology & HDD & FLASH & 3D XPoint & DRAM & STT-RAM & MRAM \\
\hline Read Latency & $5 \mathrm{~ms}$ & $100 \mu \mathrm{s}$ & $50-150 \mathrm{~ns}$ & $50 \mathrm{~ns}$ & $20 \mathrm{~ns}$ & $20 \mathrm{~ns}$ \\
Write Latency & $5 \mathrm{~ms}$ & $100 \mu \mathrm{s}$ & $50-500 \mathrm{~ns}$ & $50 \mathrm{~ns}$ & $20 \mathrm{~ns}$ & $20 \mathrm{~ns}$ \\
Endurance (Cycles) & $>10^{15}$ & $10^{4}$ & $10^{9}$ & $>10^{16}$ & $>10^{16}$ & $>10^{15}$ \\
\$ per GB & $0.025-0.5$ & $0.25-0.83$ & $6.5[33]$ & $5.3-8$ & N/A & N/A \\
Cell Size & N/A & $4-6 F^{2}$ & $4.5 F^{2}[4]$ & $10 F^{2}$ & $6-20 F^{2}$ & $25 F^{2}$ \\
\hline
\end{tabular}

Further, we note that in these new technologies writes are often more expensive that reads both in terms of latency as shown and endurance/lifetime cost, as well as energy consumption for writing.

The relative closeness in performance and capacity to traditional DRAM of emerging NVM technologies argues for a different approach to memory management than traditional, OS or hardware-cache based approaches. In the remainder of this section, we examine the prior work approaches to the design of hybrid memory systems.

\subsection{Operating System-Based Memory Man- agement}

Hassan et al., Fedorov et al. and propose to leverage the OS to manage placement and movement between NVM and DRAM $[9,10]$. They treat NVM as a parallel memory device on the same level as that of DRAM in the memory hierarchy. They argue that this approach can yield better utilization of the large NVM capacity without wasting the also relatively large DRAM capacity. Their approach is similar to the traditional approach of using storage as a swap space to extend the DRAM main memory space. Direct application of this approach to NVM creates some difficulties, however. When a given requested data is found to be in the swap space on the NVM, a page fault occurs which must be handled by operating system. The latency of this action is not only comprised of the device latency itself but also the induced OS context switch, and page fault handling. While in traditional storage systems with $m s$-level latencies, that cost is negligible, with the latency of SSD and other NVM devices significantly decreased, the OS management overheads come to dominate this latency, as discussed previously and indicated in Figure 1.

\subsection{Hardware-managed DRAM Caches and Related Approaches}

Other groups have proposed using DRAM as the cache/buffer for NVM, and thus turning DRAM into the new last level cache [29]. Similar schemes have also been applied to other memory devices with latency discrepancy in heterogeneousmemory-system(HMS). For instance, 3D-stacked DRAM was proposed as a cache for off-chip DRAM in the works $[14,21,28,34]$. A common theme in all these designs is the difficulty in lookup and maintenance of the tag storage, since the number of tags scales linearly with the cache size. Assuming the cache block size is $64 \mathrm{~B}$ and 8 bytes of tag for each block, then a 16GB DRAM cache requires $2 \mathrm{~GB}$ for the tag storage alone. That is much too large to fit in a fast, SRAM tag store. Much of the prior work explores mechanisms to shrink the tag storage overhead [22]. Some researchers explored tag reduction [11]. Others aimed to reconstruct the cache data structure. For instance, some works combine the tag or other meta-data bits into the data entry itself [14,23].

Another issue these works attempt to address is the extended latency of tag access. DRAM devices have significantly greater access latency than SRAM. Additionally, their larger cache capacity requires a longer time for the tag comparison and data selection hardware. If the requested data address misses in the TLB, it takes two accesses to the DRAM before the data can be fetched. Lee et al. attempted to avoid the tag comparison stage entirely by setting the cache block size to equal the page size, and converting virtual addresses to cache addresses directly in a modified TLB [18]. This approach, however requires several major changes to the existing system architecture including requiring extra information bits in the page table, modifying the TLB hardware and an additional global inverted page table.

Broadly, several issues exist with the previously proposed, hardware-based management techniques for future hybrid memory systems.

- As with traditional processor cache hierarchies, every memory request must go through the DRAM cache before accessing the NVM. Prior work shows that this approach is sub-optimal for systems where bandwidth is a constraint and where a parallel access path is available to both levels of memory [37]. Further, given the relatively slow DRAM access latency requiring a miss in the DRAM before accessing the NVM implies a significantly higher overall system latency.

- These works largely assume an inclusive style caching. Given the relative similarity in capacity between DRAM and NVM, this implies a significant loss of capacity.

- Given the capacities of DRAM and NVM versus SRAM used in processor caches, a traditional cache style arrangement implies a huge overhead in terms of cache meta-data. This overhead will add significant delays to the critical path of index search and tag comparison, impacting every data access.

Liu et al. propose a hardware/software, collaborative ap- 
proach to address the overheads of pure software approaches without some of the drawbacks of pure hardware caching [20]. Their approach, however, requires modifications both to the processor architecture as well as the operating system kernel. These modifications have a high NRE cost and hence is difficult to be carried out in production.

In this paper, we propose a hardware-based hybrid memory controller that is transparent to the user and as well as the operating system, thus it does not incur the overheads of management of OS based approaches. The controller is an independent module and compatible with existing hardware architectures and OSes. The controller manages both DRAM and NVM memories in flat address space to leverage the full capacity of both memory classes. Our approach also reserves a small portion of the available DRAM space to use as a hardware-managed cache to leverage spacial locality patterns seen in real application workloads to reduce writes to the NVM.

\section{Design}

Here we describe the proposed design of our proposed hardware memory management for future hybrid memory systems. Based on the discussion in Section 2 and cognizant of the characteristics of emerging NVM technologies, we aim to design a system in which the latency overheads of OS memory management are avoided, while hardware tag and meta-data overheads of traditional caching schemes are minimized.

\subsection{System Architecture Overview}

Figure 2 shows the system architecture of our proposed scheme. The data access requests are received by the Hybrid memory management unit (HMMU), if they miss in the processor cache. These are processed based on the built-in data placement policies, and forwarded with address translation to either DRAM or NVM. The HMMU also manages the migration of data between DRAM and NVM, by controlling the high-bandwidth DMA engine connecting the two types of memory devices.

\subsection{Data management policy}

A key component of the proposed HMMU design is its data management policy, i.e. the policy by which it decides where to place and when to move data between the different memory levels. Traditionally, in processor caches and elsewhere, cache blocks are managed with 64-byte lines and policies such as set-associative are used to decide what to replace upon the insertion of new lines into a given cache level. While this approach yields generally good performance results in processor caches, there are difficulties in adapting it for use in hybrid memories. As previously discussed in Section 2.3, for a hybrid memory system of 16GB comprised of 64-byte

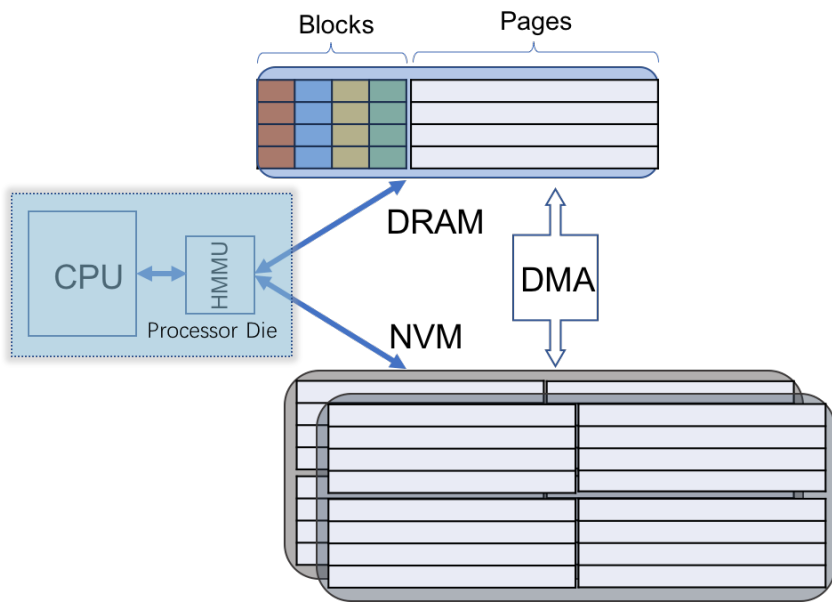

Figure 2: System Architecture Overview

cache-lines, the tag store overhead would be an impractically large 2GB. Extending the block size up to $4 \mathrm{~KB}$ to match the OS page size would significantly reduce the overheads of the tag store, bringing it down to $4 \mathrm{MB}$ for a $16 \mathrm{~GB}$ space. Since the host operating system primarily uses $4 \mathrm{~KB}$ pages, using any larger size than $4 \mathrm{~KB}$ for block management, however, risks moving a set of potentially unrelated pages together in a large block, with little, if any spatial locality between different pages in the block. This is particularly true because the addresses seen in the HMMU are "physical addresses", thus physically colocated pages may come from completely different applications, with no spatial relationship. ${ }^{1}$ As we will discuss, however, even managing blocks on a page granularity will yield greater than optimal page movements between "fast" (DRAM) and "slow" (NVM) memory levels, due to the fact that only subsets of the page are ever touched in many applications. Thus, we will examine a hybrid scheme in which most of the fast memory is managed on a page basis, lowering tag overheads, while a small fraction is managed on a sub-page basis to reduce page movement when only small portions of each page are being used at a given time.

In terms of organization and replacement, using traditional processor cache policies of set associativity and LRU replacement become unwieldy for a memory system of this size. The practical implementation of such a set-associative cache requires either a wide/multi-ported tag array (which becomes untenable for large SRAM structures) or multiple cycles to retrieve and compare each way in the set sequentially. Prior work from the OS domain [15,27] shows that, with a large number of pages to choose from, set associative, LRU replacement is not strictly necessary. Inspired by that, we first developed a simple counter-based page replacement policy.

\footnotetext{
${ }^{1}$ While many systems do allow a subset of pages to be managed at larger granularities, the HMMU has no visibility to this OS-level mapping, thus we conservatively assume $4 \mathrm{~KB}$ pages
} 


\subsubsection{Counter-based Page Management}

Rather than implementing a set associative organization with the drawbacks described above, we instead propose to implement a secondary, page-level translation table internal to the HMMU as illustrated in Figure 3. The internal page table provides a one-to-one remapping, associating each CPU-side "physical" page number in the host address space to a unique page number in the hybrid memory address space, either in the fast or slow memory. Thus, any given host page can be mapped to any location in either fast or slow memory.

While this design gives great flexibility in mapping, when a slow memory page must be moved to fast memory (i.e. upon a slow memory reference we move that page to fast memory) it requires a mechanism by which to choose the fast memory page to be replaced. Inspired by prior work in the OS domain $[15,27]$, we designed the counter-based replacement policy for this purpose.

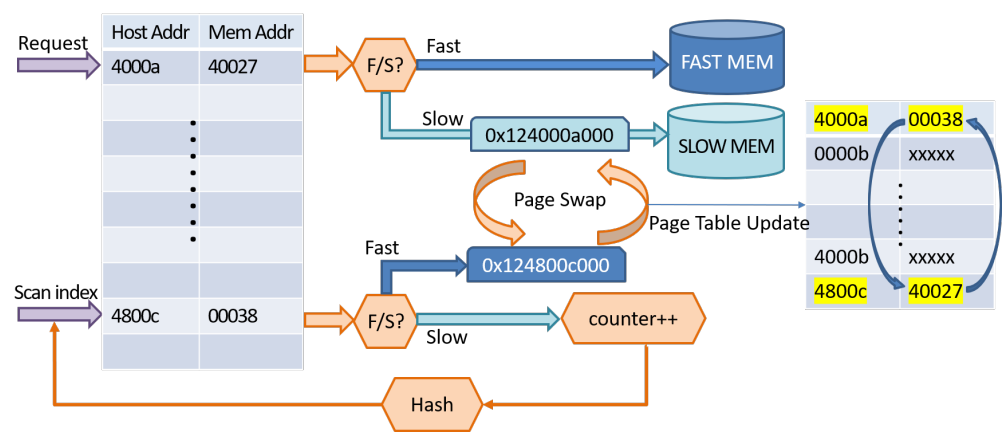

Figure 3: Counter-based Page Movement Policy

Algorithms and Design The counter-based replacement policy only requires one counter to keep track of the currently selected fast memory replacement candidate page, thus it has minimal resource overhead and can efficiently be updated each cycle. The chance that a recently accessed page gets replaced is very rare because 1 . the total number of pages is very large; 2 . the counter increases monotonically. To further reduce the possibility of evicting a recently touched page, however, we implemented a light-weight bloom filter that tracks the last 2048 accessed pages. Since checking against the bloom filter is parallel to normal page scan process, and is also executed in background, it adds no extra delay to data accesses. Algorithm 1 shows the details of the algorithm.

Figure 3 illustrates a simple example of this page movement policy. In this example memory address space, fast memory occupies internal page numbers 0 - 40000, while slow memory ranges from page number 40000 and beyond. In the figure, a request for host address 4000a arrives at the internal remapping page table. The corresponding internal page address in the memory address space is 40027 , which in this case is the 28th page in the slow memory. Here we use a policy of page movement to fast memory upon any slow memory

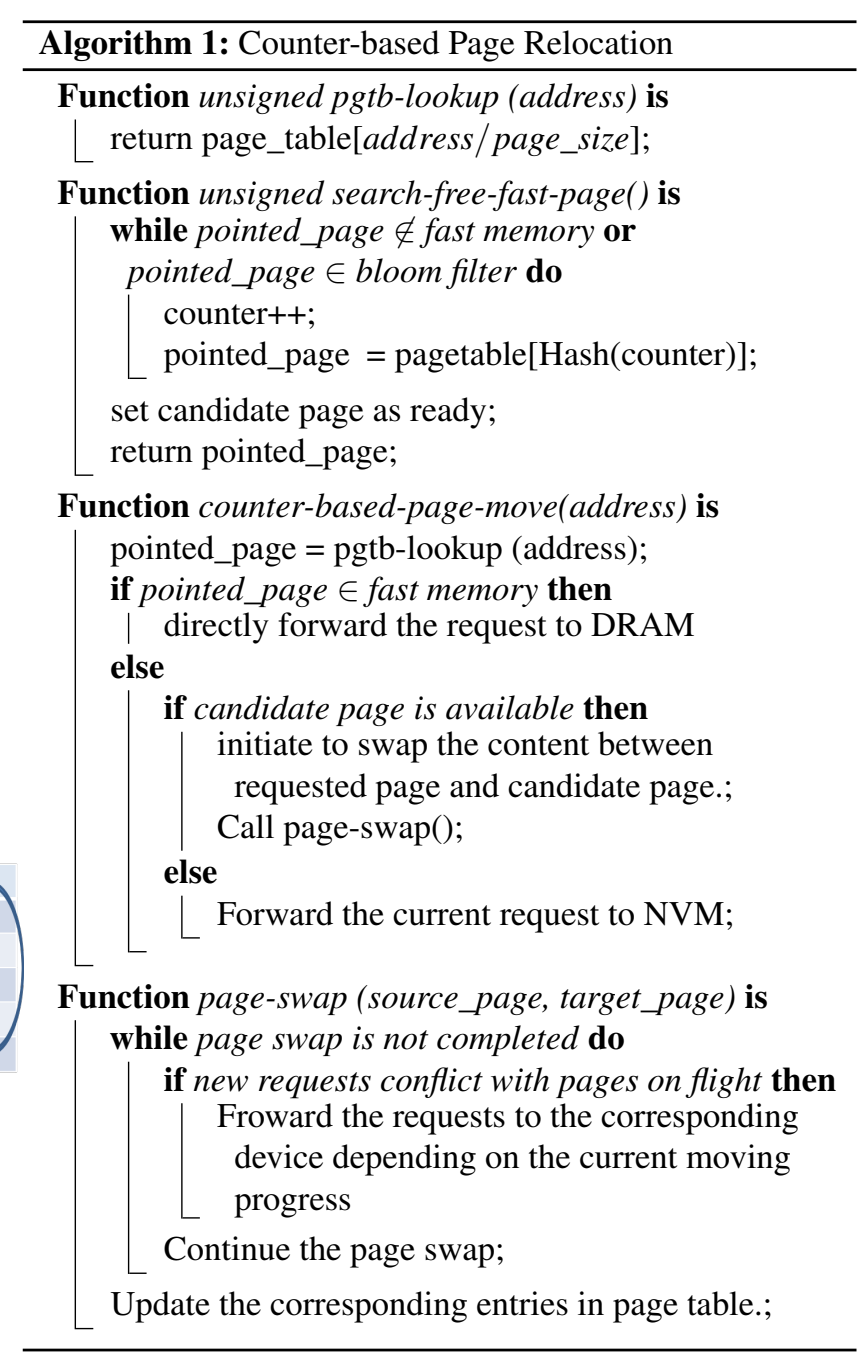

touch. $^{2}$ Thus, the HMMU directs the DMA engine to start swapping data between the requested page (40027) and the destination page in fast memory. Here, as described in Algorithm 1 the fast memory pages to be replaced is selected via the replacement counter, i.e, page 00038 in this example. Once the data swapping is completed, the memory controller updates the new memory addresses of the two swapped pages in the internal page table. Next, the counter searches for the next fast memory page replacement candidate. As the figure shows, the counter is passed through a hash function to generate an index into the internal page table. If the retrieved page number turns out to be in slow memory, the counter increments by one and the hash function generates a new index for the next query to the page table. Such process loops until it finds a page in the fast memory, which becomes the candidate destination for the page swapping.

Further details of the counter-based page management pol-

\footnotetext{
${ }^{2}$ Note that the request is serviced immediately, directly from the slow memory, while the page swap happens in the background.
} 
icy:

- Current requests are processed at top priority under all circumstances. Except for rare cases when a given write request conflicts with ongoing page movement, we always process the current request first. As for those rare cases, since all write requests are treated as non-blocking, the host system shall not suspend for them to complete. Therefore our design does not add overhead to the critical path of request processing.

- Due to the parallel nature of hardware, we search for free pages in fast memory in the background, without interference to host read request processing.

- Page-swap is initiated by the HMMU, however, it is executed by a separate DMA hardware module. Thus it does not impact other ongoing tasks.

- Data coherence and consistency are maintained during page movements.

We carefully designed the DMA process so that it could properly handle the new requests to the pages as they are being moved. All read requests and most write requests can proceed without blocking. In some very rare cases, the write requests are held until the current page copy finished.

\subsubsection{Sub-page Block Management}

Various applications could have widely different data access patterns: those with high spatial locality may access a large number of adjacent blocks of data; while others may have a larger stride between the requested addresses. For applications with weak or no spatial locality, there is very limited benefit to moving the whole page of data into fast memory, as most of the non-touched data may not be used at all. Based on this observation, we propose a scheme for sub-page size block management, which manipulates the data placement and migration in finer granularity.

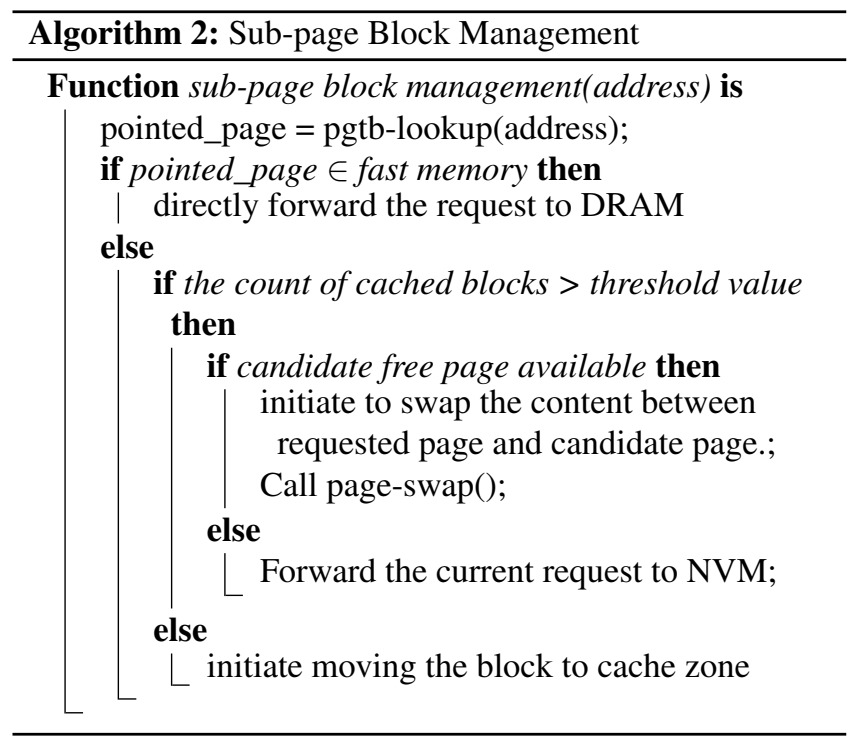

Data Migration Policy We set aside a small fraction of the fast memory and manage that area in a cache-like fashion with sub-page sized blocks. The basic algorithm used in shown in Algorithm 2. Upon the first accesses to a slow memory page, instead of moving the whole page into fast memory, we will only move the requested block of that page into the "cache" zone in fast memory. We then keep track of the total number of cached blocks belonging to every page. Only after the count of cached blocks meets a certain threshold will we swap the whole page to fast memory.

Figure 4 illustrates a simple example of the sub-page block relocation policy: The memory controller receives a request to host physical address 0x124000a200. In the first cycle, both the page table and cache metadata are checked in parallel, to decide the target memory device. If the data is found only in the slow memory, the memory controller will trigger the data relocation process. The 4-bit counter in the page table entry tells the number of sub-page blocks that have been cached for the current page. Comparing the counter against the preset threshold, determines whether to start a full-page swap or a sub-block relocation. In the given example, the counter value is 2 , which is smaller than the threshold value of 4 . Thus only that specific block containing the requested data ( $0 \times 40027200$ to $0 \mathrm{x} 4002727 \mathrm{f}$ ) will be copied to the cache. It is possible that the data might be found in both slow memory and the cache at the same time. To enforce data consistency, we always direct the read/write request to the copy in cache. This dirty data will be written back to the slow memory upon eviction.

Fast Memory Cache Design As the page size is $4 \mathrm{~KB}$, we choose 128 bytes as a reasonable block size (this size also corresponds to the DRAM open page burst size, so it sees a significant performance boost versus other block sizes). The cache is organized as a 4-way associative cache. The cache uses a pseudo-LRU as the block replacement policy. We also enable a proactive cache recycling policy: when a block is accessed, if its underlying page is detected to have been relocated to fast memory, we would evict that block from the cache to save the space for other blocks. Thus one block of data will not occupy the capacity of two copies in the fast memory at the same moment.

\subsubsection{Hardware Cost and Overhead}

Each page table entry takes $\log _{2} \frac{\text { Memory Space }}{\text { Page Size }}$ bits to represent the page address. In addition, we need some bits for statistical meta-data such as the counter of misses occurring to the page. In our sample design, the memory space is $2 \mathrm{~GB}$ and the page size is $4 \mathrm{~KB}$, thus the hardware cost per page could be rounded to $\log _{2} \frac{2 \mathrm{~GB}}{4 \mathrm{~KB}}+5 \mathrm{bits}=3$ bytes, and the total cost is $1.5 \mathrm{MB}$. The page table cost scales linearly with memory size whereas the cost per entry only grows logarithmically. The meta-data for each cache set is comprised of three parts; 


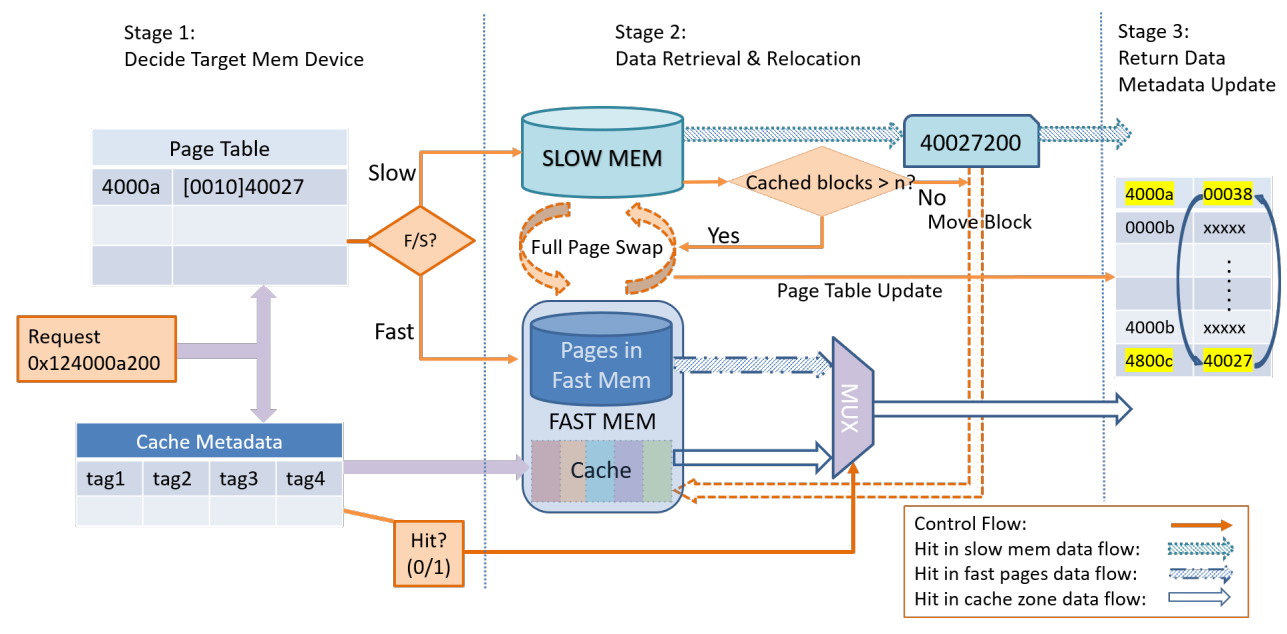

Figure 4: Sub-block Relocation Policy

four tags $(8$ bits $\times 4)$, pseudo-LRU bits (3) and dirty bits (4), which adds to 39 bits. The total cost is 39 bits $\times 2^{16} \approx 312 \mathrm{~KB}$ Since the cache is read and check parallel to the access to the page table, there is no additional timing cost for handling regular requests. The DMA provides the non-conflict data relocation for sub-page block level as same as that of the page relocation.

\subsubsection{Static versus Adaptive Caching Threshold}

With both page and block migration available, a new question arises, how to choose wisely between these two policies for optimal results. We note that these policies have different characteristics as follows:

- With page-migration, the data is exclusively placed between NVM and DRAM device. Thus larger memory space is available to applications, and the bandwidth of both devices is available.

- Sub-page-block migration is done in an inclusive cache fashion, thus avoids the additional writes to NVM when the clean data blocks are evicted from DRAM.

For applications with strong spatial locality, whole page migration maximizes performance because the migration cost is only incurred once, and the following accesses hit in the fast memory. Alternately, sub-page block promotion benefits applications with less spacial locality, because it limits writes to NVM incurred by full page migration. We further note that application behavior may vary over time with one policy being better in one phase and another better during another.

We therefore include in the page translation table an 8bit, bitmap for tracking accesses to each sub-page block of the given page. This allows measurement of the utilization rate of promoted pages. If a large portion of blocks were revisited, then we lower the threshold to allow more whole page migration. Alternately, if few blocks were accessed we suppress the whole page promotion by raising the threshold value, decreasing the rate at which full pages are migrated.

\section{Evaluation}

In this section, we present the evaluation of our proposed HMMU design. First, we present the experimental methodology. Then we discuss the performance results. Finally we analyze some of the more interesting data points.

\subsection{Methodology}

\subsubsection{Emulation Platform}

Evaluating the proposed system presents several unique challenges because we aim to test the whole system stack, comprising not only the CPU, but also the memory controller, memory devices and the interconnections. Further, since this project involves hybrid memory, accurate modeling of DRAM is required. Much of the prior work in the processor memory domain relies upon software simulation as the primary evaluation framework with tools such as Champsim [2] and gem5 [1]. However, detailed software simulators capable of our goals impose huge simulation time slow-downs versus real hardware. Furthermore, there are often questions of the degree of fidelity of the outcome of arbitrary additions to software simulators [26].

Another alternative used by some prior work [9] is to use an existing hardware system to emulate the proposed work. This method could to some extent alleviate the overlong the simulation runtime, however, no existing system supports our proposed HMMU.

Thus, we elected to emulate the HMMU architecture on an FPGA platform. FPGAs provide flexibility to develop and test sophisticated memory management policies while its hardware-like nature provides near-native simulation speed. The FPGA communicates with the ARM CortexA57 CPU via 
a high-speed PCI Express link, and manages the two memory modules(DRAM and NVM) directly. The DRAM and NVM memories are mapped to the physical memory space via the PCI BAR(Base Address Register) window. From the perspective of the CPU, they are rendered as available memory resource same as other regions of this unified space.

Our platform emulates various NVM access delays by adding stall cycles to the operations executed in FPGA to access external DRAM. The platform is not constrained to any specific type of NVM, but rather allows us to study and compare the behaviors across any arbitrary combinations of hybrid memories. In the following sections, we would show the simulation results with different memory devices. The detailed system specification is listed in Table 2.

Table 2: Emulation System Specification

\begin{tabular}{l|l}
\hline Component & Description \\
\hline CPU & $\begin{array}{l}\text { ARM Cortex-A57 @ 2.0GHz, 8 cores, ARM v8 } \\
\text { architecture }\end{array}$ \\
\hline L1 I-Cache & 48 KB instruction cache, 3-way set-associative \\
\hline L1 D-Cache & 32 KB data cache, 2-way set-associative \\
\hline L2 Cache & 1MB, 16-way associative, 64kB cache line size \\
\hline $\begin{array}{l}\text { Interconnec- } \\
\text { tion }\end{array}$ & PCI Express Gen3 (8.0 Gbps) \\
\hline Memory & 128MB DDR4 + 1GB NVM \\
\hline OS & Linux version 4.1.8 \\
\hline
\end{tabular}

We measured the round trip time in FPGA cycles to access external DRAM DIMM first, and then scaled the number of stalled cycles according to the speed ratio between DRAM and future NVM technologies, as described in Section 2.1. Thus we have one DRAM DIMM running at full speed and the other DRAM DIMM emulating the approximate speed of NVM Memory.

\subsubsection{Workloads}

We initially considered several mobile-specific benchmark suites, including the CoreMark [32] and AndEBench [6] from EEMBC. We found however that these suites are largely out of date and do not accurately represent the large application footprints found on modern mobile systems. Also, in some cases they are only available as closed source [6] and thus are unusable in our infrastructure. Instead, we use applications from the recently released SPEC CPU 2017 benchmark suite [35]. To emulate memory intensive workloads for future mobile space, we selected only those SPEC CPU 2017 benchmarks which require a larger working set than the fast memory size in our system. The details of tested benchmarks are listed in Table 3.

To ensure that application memory was allocated to the HMMU's memory, the default Linux malloc functions are replaced with a customized jemalloc [8]. Thus the HMMU memory access was transparent to the CPU and cache, and no benchmark changes were needed.
Table 3: Tested Workloads [35]

\begin{tabular}{l|l|l}
\hline Benchmark & Description & Memory footprint \\
\hline \multicolumn{3}{|c}{ Integer Application } \\
\hline 500.perlbench & Perl interpreter & 202MB \\
\hline 520.omnetpp & $\begin{array}{l}\text { Discrete Event simulation - } \\
\text { computer network }\end{array}$ & $241 \mathrm{MB}$ \\
\hline 523.xalancbmk & $\begin{array}{l}\text { XML to HTML conversion via } \\
\text { XSLT }\end{array}$ & $481 \mathrm{MB}$ \\
\hline 531.deepsjeng & $\begin{array}{l}\text { Artificial Intelligence: } \\
\text { alpha-beta tree search (Chess) }\end{array}$ & 700MB \\
\hline 557.xz & General data compression & 727MB \\
\hline \multicolumn{3}{|c}{ Float Point Application } \\
\hline 510.parest & $\begin{array}{l}\text { Biomedical imaging: optical } \\
\text { tomography with finite } \\
\text { elements }\end{array}$ & $413 \mathrm{MB}$ \\
\hline 519.1bm & Fluid dynamics & $410 \mathrm{MB}$ \\
\hline 538.imagick & Image Manipulation & $287 \mathrm{MB}$ \\
\hline 544.nab & Molecular Dynamics & $147 \mathrm{MB}$
\end{tabular}

\subsubsection{Designs Under Test}

Here we test the following data management policies developed for use with our HMMU:

- Static: A baseline policy in which host requested pages are randomly assigned to fast and slow memory. This serves as a nominal, worst-case, memory performance.

- PageMove: The whole 128MB DRAM is manged on the granularity of $4 \mathrm{k}$ pages. When a memory request is missed in fast memory, the DMA engine will trigger a page relocation from slow memory to fast memory, as described in Section 3.2.1.

- StatComb: Here 16MB out of the 128MB DRAM is reserved for sub-page block relocation, managed in the cache-like fashion, as described in Section 3.2.2. The remainder of the DRAM is managed on a full page basis. An empirically derived static threshold of 4 blocks touched is used to determine when a full page should be moved to the page portion of DRAM.

- AdpComb: Same as StatComb, except that, as described in Section 3.2.4, an adaptive threshold is used to determine when the full page should be moved.

- AIIDRAM: Here we implement a baseline policy in which there is sufficient fast memory to serve all pages in the system and no page movement is required. This serves as a nominal, best-case but impractical memory performance design.

\subsection{Results}

\subsubsection{Energy Saving}

Emerging NVM consumes minimal standby power, which could help save energy consumption on mobile computation. We evaluated and compared the energy spent in running SPEC 2017 benchmarks between the full DRAM configuration and our policies. We referred to Micron DDR4 technical spec [24] 
for DRAM and recent work on 3DxPoint [17] for NVM device power consumption, respectively (Table 4).

Table 4: Power Consumption of DDR4 and 3D-XPoint

\begin{tabular}{c|c|c}
\hline Technology & DDR4 & 3Dxpoint \\
\hline Read Latency & $50 \mathrm{~ns}$ & $100 \mathrm{~ns}$ \\
Write Latency & $50 \mathrm{~ns}$ & $300 \mathrm{~ns}$ \\
Read Energy & $4.2 \mathrm{~nJ}$ & $1.28 \mathrm{~nJ}$ \\
Write Energy & $3.5 \mathrm{~nJ}$ & $8.7 \mathrm{~nJ}$ \\
Background Power & $30 \mathrm{~mW} / \mathrm{GB}$ & $\sim 0$ \\
\hline
\end{tabular}

We normalize the energy consumption of our policies to that of the AllDRAM configuration and present them in the figure 5. In the figure we see that all three techniques save a

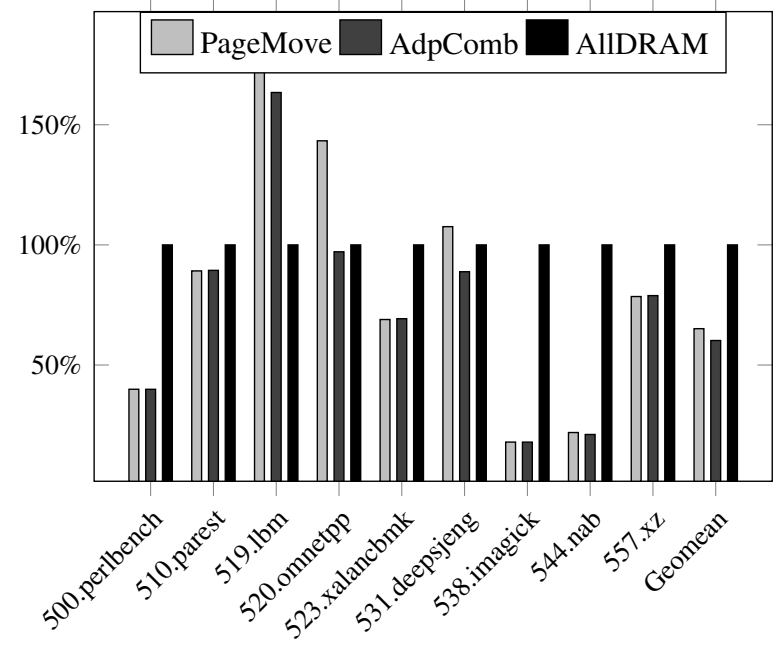

Figure 5: Energy Consumption Comparison

substantial amount of energy. On average the AdpComb adaptive policy only consumes $60.2 \%$ energy as compared to the AllDRAM configuration, while the PageMove and StatComb policies are at $65.1 \%$ and $63.6 \%$, respectively. That said, several benchmarks see energy consumption increases under the PageMove policy, while StatComb, sees a significant regression in energy consumption for 519.1bm. AdpComb, while also seeing increased energy consumption under 519.1bm, shows better energy consumption than the other two policies for nearly all cases.

Further investigating the distribution of energy consumption, we track the DRAM background power, number of DRAM read/writes and NVM read/writes. We present the comparison between AdpComb and AllDRAM in Figure 6. Since $7 / 8$ of the memory was replaced with NVM, the standby power shrinks significantly. Although write operations to NVM dissipate more energy than DRAM, the AdpComb policy avoids most of this increase by absorbing many writes in DRAM. Our policies saw the greatest energy efficiency improvement with applications imagick and nab, which spent

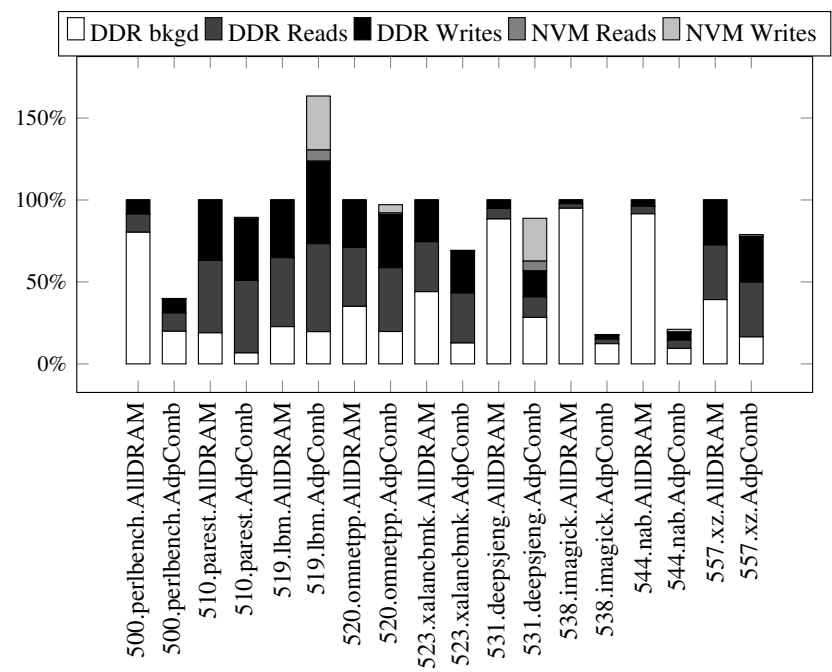

Figure 6: Energy Consumption Breakdown

$17.9 \%$ and $21.1 \%$ energy compared to full DRAM. We find that these two applications have high processor cache hit rates and spent most time in computation. Thus they have few references to the memory, and the largest portion of energy was spent on DRAM background power. Thus AptComb policy's advantage of having much lower DRAM static power is best exploited. Our policies did pretty well with all benchmark applications except $\mathrm{lbm}$, which spent $63 \%$ more energy. This application incurred a massive number of cache block writebacks to NVM. We investigated the case and found $\mathrm{lbm}$ has the highest percentage of store instructions among all benchmark applications [19]. This creates many dirty blocks, and thus writebacks are expected when blocks are later evicted. The amount of writes is also amplified by the writebacks of cache blocks.

\subsubsection{Runtime Performance}

Figure 7 shows the speedup attained by the different designs under test for the various benchmarks in the SPEC CPU 2017 benchmark suite. Here all the results are normalized to the runtime of the ideal, AllDRAM, DRAM configuration. We see that the average performance of AdpComb is $88.4 \%$, while the random static allocation "Static" only yields $40 \%$ of the full DRAM performance. Thus, the adaptive policy achieves more than $2 \mathrm{x}$ performance benefit versus the worst-case, static allocation policy under the same memory resource. Generally the AdpComb policy outperforms the other two policies we propose, though interestingly, for many benchmarks, including perlbench, parest, xalancbmk, xz, imagick, and nab, PageMove comes within 5\% of the performance of AllDRAM. 


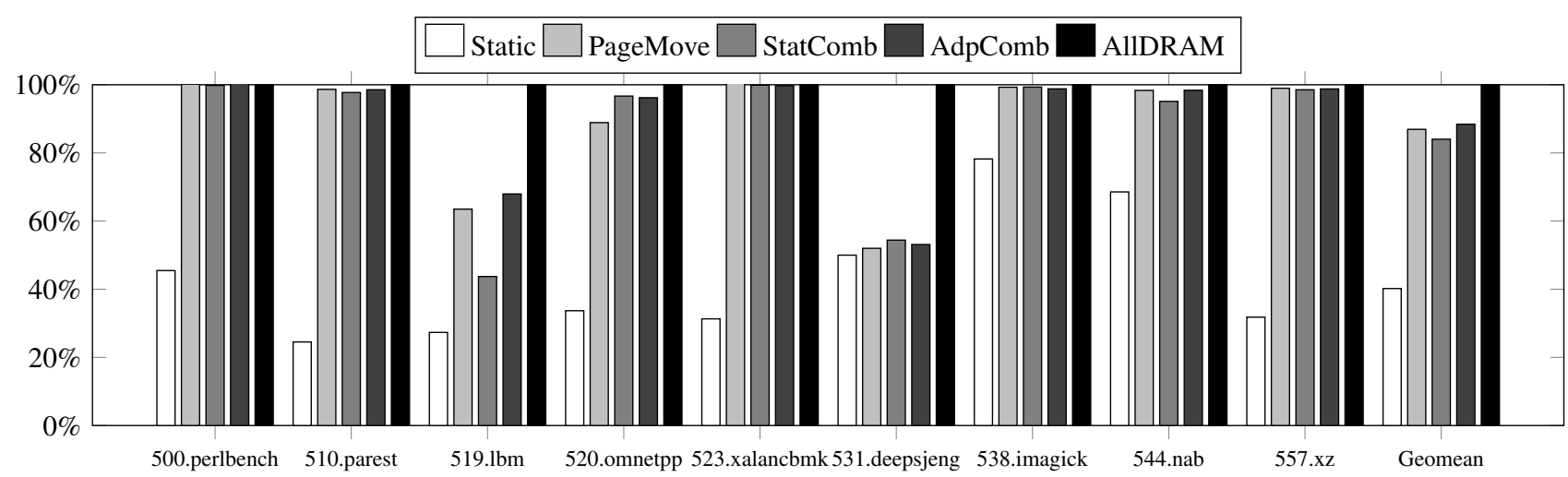

Figure 7: SPEC 2017 Performance Speedup

\subsection{Analysis and Discussion}

The adaptive AdpComb policy successfully reduces energy by $40 \%$, with a modest $12 \%$ loss of the performance versus an unrealistic and unscalable AllDRAM design. AdpComb attempts to make the optimal choice between the PageMove and the StatComb block migration policy. In the remainder of this text, we will further analyze the experiment results.

\subsubsection{PageMove Policy Performance}

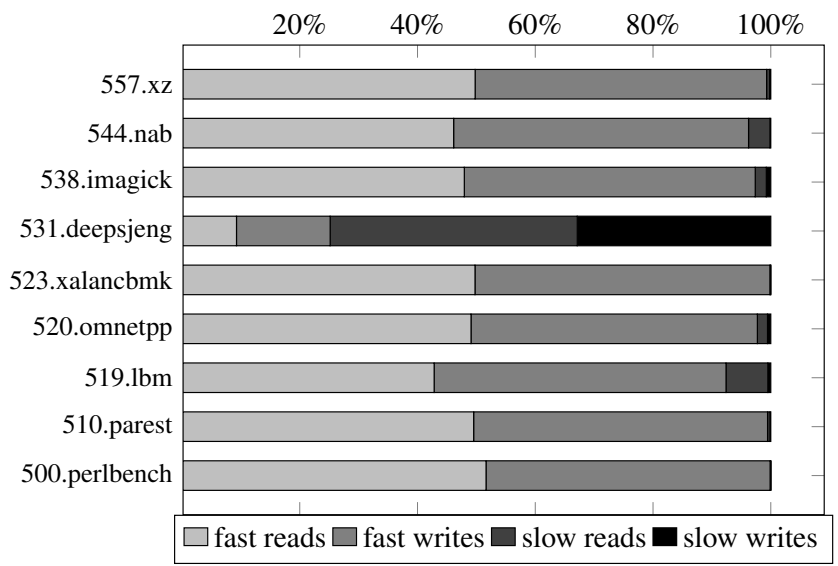

Figure 8: Memory Accesses Breakdown of PageMove Policy

The PageMove policy has similar average runtime performance $(86.9 \%)$ to the adaptive AdpComb policy (88.4\%). Figure 8 shows the breakdown of memory requests that hit in the fast pages and slow pages respectively. When compared to the speedup in Figure 7, we see the benchmarks which PageMove policy works best have most of their memory requests hitting in the fast pages, while the hit rate in slow pages become negligible. This provides a large performance boost considering that the system's slow memory is $8 \mathrm{x}$ slower than the fast memory.
In the figure, the StatComb policy has an overall speedup of $84 \%$ against the AllDRAM configuration. The difference is mainly contributed by 519.1bm and 544.nab. As we will show, however, StatComb does still provide significant benefits in terms of total writes to NVM.

The PageMove policy performs worst on the benchmark 531.deepsjeng, with a slowdown of 52\% versus AllDRAM. We divided the number of hits in fast memory by the occurrences of page relocation, and found that deepsjeng has the lowest rate (0.03) across all the benchmark applications (Geomean is 3.96). This suggests that when a page is relocated from slow memory to fast memory, the remainder of that page is often not extensively utilized. Further, we also see an exceptionally high ratio of blocks moved to cache versus page relocation. The geometric mean of all benchmarks is 10.5 while deepsjeng marks 397 . This is a sign that in most cases, the page is only visited for one or two lines, and never accumulates enough cached blocks to begin a whole page relocation. To sum up, deepsjeng has a sparse and wide-range memory access pattern, which is quite difficult to prefetch effective data or improve performance.

$519.1 \mathrm{bm}$ presents another interesting case, since its performance is also poor. Similar to deepsjeng, the hit rate in fast memory is low in contrast to the number of page relocations. However, a key difference is that over $60 \%$ of the cached blocks were evicted after its underlying pages relocated to fast memory. This indicates that $\mathrm{lbm}$ walks through many blocks of the same page and triggers the whole page relocation quickly. On that account, we deduce that this benchmark will benefit from a configuration with more fast pages and a smaller cache zone. We reran this benchmark with a cache size of $8 \mathrm{MB}$ and the threshold value of 1 , and found a supportive result of $8 \%$ performance gain on top of the default threshold value of 4 .

\subsubsection{Writes Reduction and NVM lifetime Saving}

Unlike the traditional DRAM, emerging NVM technologies have different characteristics for reads and writes. Write oper- 


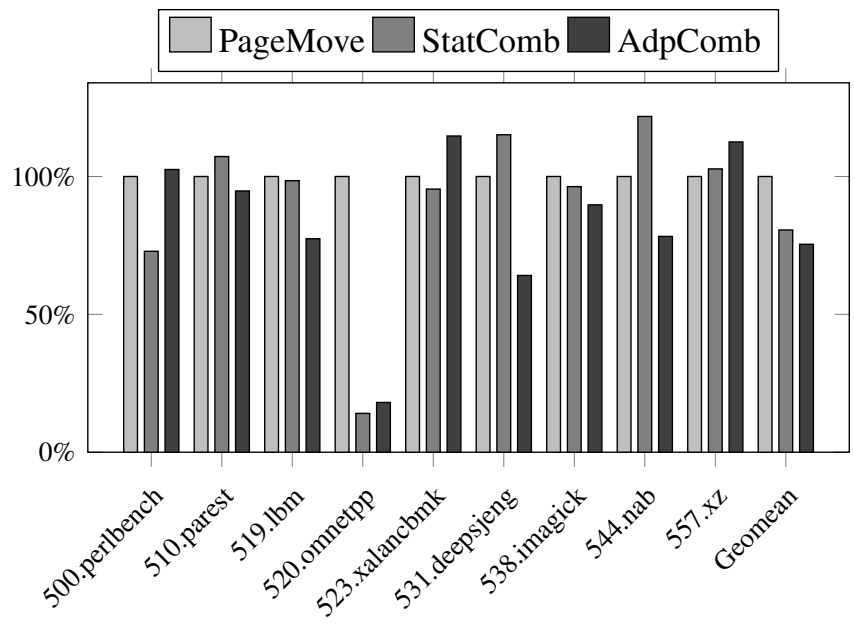

Figure 9: Writes to NVM

ations dissipates more than $8 \mathrm{x}$ the energy of reads [3]. Moreover, NVM technologies often have limited write endurance, i.e, the maximum cycles of writes before they wear out. Hence, if we could reduce the amount of writes, we could greatly save energy consumption and extend the lifetime of NVM device. Figure 9 shows the percentage of writes to slow memory for both techniques, normalized against the number of writes seen in the PageMove policy. Please note that we measure not only the direct writes from the host but also the writes induced by page movements and sub-page block writebacks to slow memory. In the figure we see that our combined policy has an average of $20 \%$ fewer writes than the PageMove policy. While several benchmarks benefit from the sub-page block cache, this advantage is strongest with omnetpp, with a drop of $86 \%$. The detailed analysis of this particular benchmark is presented in the next section.

\subsubsection{Sensitivity to Threshold}

The extraordinary reduction of writes for omnetpp is intriguing. We reran the tests with different StatComb static page relocation thresholds and examined the changes in run time and total numbers of writes to NVM. In Figure 10, we normalized all numbers to the value for a threshold of 4 , the threshold used in StatComb. The runtime varied according to the same trend as the number of writes, and the threshold value of 4 turned out to be the overall sweet spot. Both metrics started to deteriorate rapidly when the threshold value shifted. Then we measured the number of writes to NVM incurred by page relocation and block relocation, respectively. The results represented by stacked bars, reveals the reason why threshold of 4 is the best choice: More pages were relocated when the threshold was lowered. On the other hand, the amount of block migration grew rapidly as the threshold increased. The trade-offs reached perfect balance at the value of 4 , which had a slightly more page moves than that of value

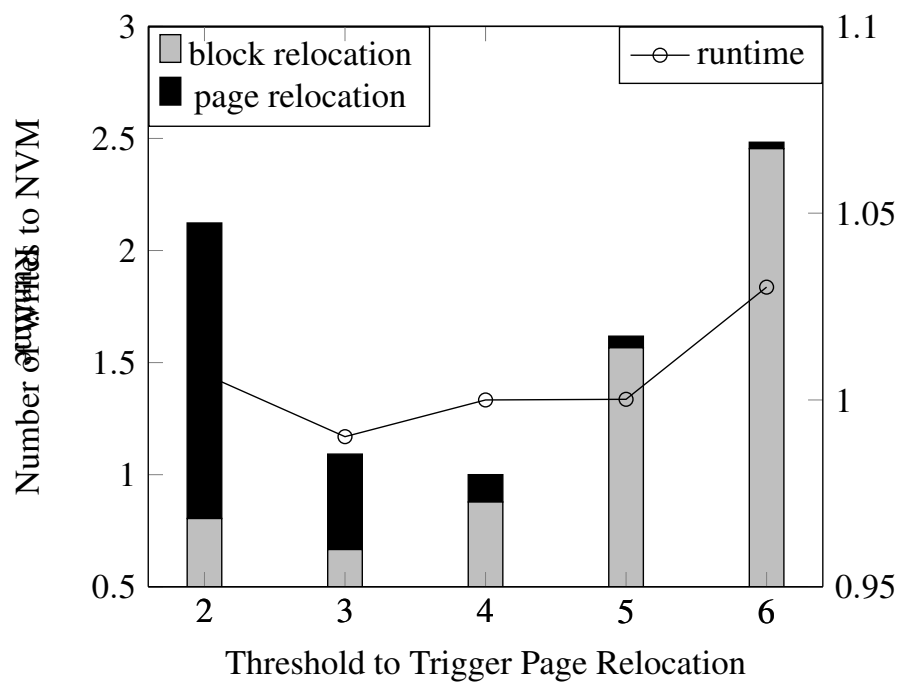

Figure 10: Omnetpp Performance Analysis

5 , yet significantly fewer block migrations.

\subsubsection{Adaptive Policy}

The analysis above showed that the whole page promotion policy favors certain benchmark applications, in which most blocks were revisited on the promoted pages. Meanwhile other applications benefit from sub-page block promotions as only a subset of blocks were re-utilized. If we could always choose the correct policy for each application, then we could expect the optimal results for overall performance. These results reinforce the reasoning behind our AdpComb policy's adaptive threshold, wherein for applications where pages are mostly utilized full page movement is completed quickly, while for applications where accesses are sparse, page movement is postponed till most of the page has been touched once.

\section{Conclusions}

A wide spectrum of non-volatile memory (NVM) technologies are emerging, including phase-change memories (PCM), memristor, and 3D XPoint. These technologies look particularly appealing for inclusion in the mobile computing memory hierarchy. While NVM provides higher capacity and less static power consumption, than traditional DRAM, its access latency and write costs remain problematic. Integration of these new memory technologies in the mobile memory hierarchy requires a fundamental rearchitecting of traditional system designs. Here we presented a hardware-accelerated memory manager that addresses both types of memory in a flat space address space. We also designed a set of data placement and data migration policies within this memory manager, such that we may exploit the advantages of each 
memory technology. While the page move policy provided good performance, adding a sub-page-block caching policy helps to reduce writes to NVM and save energy. On top of these two fundamental policies, we built an adaptive policy that intelligently chooses between them, according to the various phases of the running application. Experimental results show that our adaptive policy can significantly reduce power consumption by almost $40 \%$. With only a small fraction of the system memory implemented in DRAM, the overall system performance comes within $12 \%$ of the full DRAM configuration, which is more than $2 \mathrm{X}$ the performance of random allocation of NVM and DRAM. By reducing the number of writes to NVM, our policy also helps to extend device lifetime.

\section{References}

[1] Nathan Binkert, Bradford Beckmann, Gabriel Black, Steven K. Reinhardt, Ali Saidi, Arkaprava Basu, Joel Hestness, Derek R. Hower, Tushar Krishna, Somayeh Sardashti, Rathijit Sen, Korey Sewell, Muhammad Shoaib, Nilay Vaish, Mark D. Hill, and David A. Wood. The gem5 simulator. SIGARCH Comput. Archit. News, 39(2):1-7, August 2011.

[2] ChampSim. Champsim, 2016. https://github.com/ Champsim/Champsim.

[3] An Chen. A review of emerging non-volatile memory (nvm) technologies and applications. Solid-State Electronics, 125:25-38, 2016.

[4] Jeongdong Choe. Intel 3d xpoint memory die removed from intel optane pcm, 2017. https://www. techinsights.com/blog/intel-3dxpoint-memory-die-removed-intel-optanetmpcm-phase-change-memory.

[5] C. C. Chou, A. Jaleel, and M. K. Qureshi. Cameo: A two-level memory organization with capacity of main memory and flexibility of hardware-managed cache. In 2014 47th Annual IEEE/ACM International Symposium on Microarchitecture, pages 1-12, Dec 2014.

[6] EEMBC. An eembc benchmark for android devices, 2015. http: / / www . eembc.org/andebench.

[7] K. Eshraghian, Kyoung-Rok Cho, O. Kavehei, Soon-Ku Kang, D. Abbott, and Sung-Mo Steve Kang. Memristor mos content addressable memory (mcam): Hybrid architecture for future high performance search engines. Very Large Scale Integration (VLSI) Systems, IEEE Transactions on, 19(8):1407-1417, Aug 2011.

[8] Jason Evans. Jemalloc, 2016. http://jemalloc. net/.
[9] Viacheslav Fedorov, Jinchun Kim, Mian Qin, Paul V. Gratz, and A. L. Narasimha Reddy. Speculative paging for future nvm storage. In Proceedings of the International Symposium on Memory Systems, MEMSYS '17, pages 399-410, New York, NY, USA, 2017. ACM.

[10] Ahmad Hassan, Hans Vandierendonck, and Dimitrios S. Nikolopoulos. Software-managed energy-efficient hybrid dram/nvm main memory. In Proceedings of the 12th ACM International Conference on Computing Frontiers, CF'15, pages 23:1-23:8, New York, NY, USA, 2015. ACM.

[11] Cheng-Chieh Huang and Vijay Nagarajan. Atcache: Reducing dram cache latency via a small sram tag cache. In Proceedings of the 23rd International Conference on Parallel Architectures and Compilation, PACT'14, pages 51-60, New York, NY, USA, 2014. ACM.

[12] INTEL CORPORATION. Intel 750, 2015. https://ark.intel.com/products/86740/IntelSSD-750-Series-400GB-12-Height-PCIe-3_0$20 \mathrm{~nm}-\mathrm{MLC}$.

[13] INTEL CORPORATION. Intel optane technology, 2016. https://www.intel.com/content/www/ us/en/architecture-and-technology/inteloptane-technology.html.

[14] D. Jevdjic, G. H. Loh, C. Kaynak, and B. Falsafi. Unison cache: A scalable and effective die-stacked dram cache. In 2014 47th Annual IEEE/ACM International Symposium on Microarchitecture, pages 25-37, Dec 2014.

[15] Theodore Johnson and Dennis Shasha. 2q: A low overhead high performance buffer management replacement algorithm. In $V L D B, 1994$.

[16] Youngjin Kwon, Henrique Fingler, Tyler Hunt, Simon Peter, Emmett Witchel, and Thomas Anderson. Strata: A cross media file system. In Proceedings of the 26th Symposium on Operating Systems Principles, SOSP '17, pages 460-477, New York, NY, USA, 2017. ACM.

[17] Benjamin C. Lee, Engin Ipek, Onur Mutlu, and Doug Burger. Architecting phase change memory as a scalable dram alternative. In Proceedings of the 36th Annual International Symposium on Computer Architecture, ISCA '09, pages 2-13, New York, NY, USA, 2009. ACM.

[18] Y. Lee, J. Kim, H. Jang, H. Yang, J. Kim, J. Jeong, and J. W. Lee. A fully associative, tagless dram cache. In 2015 ACM/IEEE 42nd Annual International Symposium on Computer Architecture (ISCA), pages 211-222, June 2015. 
[19] A. Limaye and T. Adegbija. A workload characterization of the spec cpu2017 benchmark suite. In 2018 IEEE International Symposium on Performance Analysis of Systems and Software (ISPASS), pages 149-158, 2018.

[20] Haikun Liu, Yujie Chen, Xiaofei Liao, Hai Jin, Bingsheng He, Long Zheng, and Rentong Guo. Hardware/software cooperative caching for hybrid dram/nvm memory architectures. In Proceedings of the International Conference on Supercomputing, ICS '17, pages 26:1-26:10, New York, NY, USA, 2017. ACM.

[21] N. Madan, L. Zhao, N. Muralimanohar, A. Udipi, R. Balasubramonian, R. Iyer, S. Makineni, and D. Newell. Optimizing communication and capacity in a $3 \mathrm{~d}$ stacked reconfigurable cache hierarchy. In 2009 IEEE 15th International Symposium on High Performance Computer Architecture, pages 262-274, Feb 2009.

[22] J. Meza, J. Chang, H. Yoon, O. Mutlu, and P. Ranganathan. Enabling efficient and scalable hybrid memories using fine-granularity dram cache management. IEEE Computer Architecture Letters, 11(2):6164, 2012.

[23] J. Meza, J. Chang, H. Yoon, O. Mutlu, and P. Ranganathan. Enabling efficient and scalable hybrid memories using fine-granularity dram cache management. IEEE Computer Architecture Letters, 11(2):6164, 2012.

[24] Inc. Micron Technology. Calculating memory power for ddr4 sdram. Technical report, 2017.

[25] S. Mittal and J. S. Vetter. A survey of software techniques for using non-volatile memories for storage and main memory systems. IEEE Transactions on Parallel and Distributed Systems, 27(5):1537-1550, 2016.

[26] T. Nowatzki, J. Menon, C. Ho, and K. Sankaralingam. Architectural simulators considered harmful. IEEE Micro, 35(6):4-12, Nov 2015.

[27] Elizabeth J. O'Neil, Patrick E. O'Neil, and Gerhard Weikum. The lru-k page replacement algorithm for database disk buffering. In Proceedings of the 1993 ACM SIGMOD International Conference on Management of Data, SIGMOD '93, pages 297-306, New York, NY, USA, 1993. ACM.

[28] M. K. Qureshi and G. H. Loh. Fundamental latency trade-off in architecting dram caches: Outperforming impractical sram-tags with a simple and practical design. In 2012 45th Annual IEEE/ACM International Symposium on Microarchitecture, pages 235-246, Dec 2012.
[29] Moinuddin K. Qureshi, Vijayalakshmi Srinivasan, and Jude A. Rivers. Scalable high performance main memory system using phase-change memory technology. In Proceedings of the 36th Annual International Symposium on Computer Architecture, ISCA '09, pages 24-33, New York, NY, USA, 2009. ACM.

[30] S. Raoux, G. W. Burr, M. J. Breitwisch, C. T. Rettner, Y. . Chen, R. M. Shelby, M. Salinga, D. Krebs, S. . Chen, H. . Lung, and C. H. Lam. Phase-change random access memory: A scalable technology. IBM Journal of Research and Development, 52(4.5):465-479, July 2008.

[31] INTERNATIONAL TECHNOLOGY ROADMAP FOR SEMICONDUCTORS. Moremoore, 2015. https: //www. semiconductors.org/resources/2015international-technology-roadmap-forsemiconductors-itrs/.

[32] Markus Levy Shay Gal-On. Exploring coremark - a benchmark maximizing simplicity and efficacy, 2012. https://www. eembc.org/techlit/ articles/coremark-whitepaper.pdf.

[33] Anton Shilov. Pricing of intel's optane dc persistent memory modules, 2019. https://www anandtech. com/show/14180/pricing-of-intels-optane-dcpersistent-memory-modules-leaks.

[34] J. Sim, A. R. Alameldeen, Z. Chishti, C. Wilkerson, and H. Kim. Transparent hardware management of stacked dram as part of memory. In 2014 47th Annual IEEE/ACM International Symposium on Microarchitecture, pages 13-24, Dec 2014.

[35] SPEC. SPEC CPU2017 Documentation, 2017. https: //www. spec.org/cpu2017/Docs/.

[36] Z. Wang, Z. Gu, and Z. Shao. Optimizated allocation of data variables to $\mathrm{pcm} / \mathrm{dram}$-based hybrid main memory for real-time embedded systems. IEEE Embedded Systems Letters, 6(3):61-64, Sept 2014.

[37] X. Wu and A. L. N. Reddy. Managing storage space in a flash and disk hybrid storage system. In 2009 IEEE International Symposium on Modeling, Analysis Simulation of Computer and Telecommunication Systems, pages $1-4$, Sept 2009.

[38] J. Joshua Yang, Dmitri B. Strukov, and Duncan R. Stewart. Memristive devices for computing. Nature Nanotechnology, Dec 2012.

[39] Jie Zhang, Miryeong Kwon, Donghyun Gouk, Sungjoon Koh, Changlim Lee, Mohammad Alian, Myoungjun Chun, Mahmut Taylan Kandemir, Nam Sung Kim, Jihong Kim, and Myoungsoo Jung. Flashshare: Punching through server storage stack from kernel to firmware for 
ultra-low latency ssds. In 13th USENIX Symposium on Operating Systems Design and Implementation (OSDI
18), pages 477-492, Carlsbad, CA, 2018. USENIX Association. 